\title{
Author Correction: The Methylation Capacity of Arsenic and Insulin Resistance are Associated with Psychological Characteristics in Children and Adolescents
}

\section{Ying-Chin Lin ${ }^{1,2,3}$, Chien-Tien Su ${ }^{4,5}$, Horng-Sheng Shive ${ }^{6}$, Wei-Jen Chen ${ }^{5}$, Yi-Hua Chen ${ }^{5}$, Cheuk-Sing Choy, ${ }^{7,8}$, Hung-Yi Chiou ${ }^{5}$, Bor-Cheng Han ${ }^{5}$ \& Yu-Mei Hsueh ${ }^{4,9}$}

Correction to: Scientific Reports https://doi.org/10.1038/s41598-017-03084-2, published online 08 June 2017

The original version of this Article contained an error in Affiliation 3, which was incorrectly given as 'Division of Family Medicine, School of Medicine, Taipei Medical University, Taipei, Taiwan'. The correct affiliation is listed below:

Department of Family Medicine, School of Medicine, College of Medicine, Taipei Medical University, Taipei, Taiwan

This error has now been corrected in the HTML and PDF versions of the Article.

(a) Open Access This article is licensed under a Creative Commons Attribution 4.0 International (c) License, which permits use, sharing, adaptation, distribution and reproduction in any medium or format, as long as you give appropriate credit to the original author(s) and the source, provide a link to the Creative Commons license, and indicate if changes were made. The images or other third party material in this article are included in the article's Creative Commons license, unless indicated otherwise in a credit line to the material. If material is not included in the article's Creative Commons license and your intended use is not permitted by statutory regulation or exceeds the permitted use, you will need to obtain permission directly from the copyright holder. To view a copy of this license, visit http://creativecommons.org/licenses/by/4.0/.

(C) The Author(s) 2018

\footnotetext{
${ }^{1}$ Department of Family Medicine, Shung Ho Hospital, Taipei Medical University, Taipei, Taiwan. ${ }^{2}$ Department of Health Examination, Wan Fang Hospital, Taipei Medical University, Taipei, Taiwan. ${ }^{3}$ Department of Family Medicine, School of Medicine, College of Medicine, Taipei Medical University, Taipei, Taiwan. ${ }^{4}$ Department of Family Medicine, Taipei Medical University Hospital, Taipei, Taiwan. ${ }^{5}$ School of Public Health, College of Public Health, Taipei Medical University, Taipei, Taiwan. ${ }^{6}$ Department of Chinese Medicine, Chang Gung Memorial Hospital, and Chang Gung University College of Medicine, Taoyuan, Taiwan. ${ }^{7}$ Emergency Department, Taipei Hospital, Ministry of Health and Welfare, New Taipei City, Taiwan. ${ }^{8}$ Department of Medicine, School of Medicine, College of Medicine, Taipei Medical University, Taipei, Taiwan. ${ }^{9}$ Department of Public Health, School of Medicine, College of Medicine, Taipei Medical University, Taipei, Taiwan. Correspondence and requests for materials should be addressed to B.-C.H. (email: bchan@tmu.edu.tw) orY.-M.H. (email:ymhsueh@tmu.edu.tw)
} 